\title{
Dispositif Hybride De Formation Universitaire Des Enseignants : Etude De Cas
}

\author{
Dr. Samar Zeitoun \\ Dr. Rima Malek \\ Université Libanaise, Faculté de Pédagogie, Lebanon
}

\section{Doi:10.19044/esj.2018.v14n28p15 URL:http://dx.doi.org/10.19044/esj.2018.v14n28p15}

\begin{abstract}
The adoption of blended learning is an under-researched process in the Lebanese academic context, especially when students are teachers in professional development. Although the movement towards blended learning is generally initiated in some university courses, little research has investigated teachers' perceptions of the factors that determine their success and minimize resistance, especially at the level of delivery. This article explores the adoption of hybrid training in a course at the Lebanese university. This case study explores, based on the questionnaires administered to a sample of 65 trainee teachers, the concept of blended learning from their points of view. The questionnaire included five main lines based on the literature such as (a) learning process, (b) trainer support, (c) equipment, (d) collaboration and (e) technology. The results indicated that teachers were satisfied with both their participation in the course and the knowledge gained during the training as well. The results also revealed a number of factors to consider when designing such courses in the Lebanese context.
\end{abstract}

Keywords: Blended training; Lebanon; University education; education technology, professional development, professional development, inservice teachers.

\section{Résumé}

L'adoption de l'apprentissage mixte est un processus peu recherché dans le contexte universitaire libanais surtout quand les étudiants sont des enseignants en formation continue. Bien que le mouvement vers l'apprentissage mixte soit généralement initié au sein de quelques cours universitaires, peu de recherches ont investigué les perceptions des enseignants vis-à-vis des facteurs qui déterminent son succès et minimisent la résistance surtout au niveau de la prestation. Cet article explore l'adoption d'une formation hybride dans un cours à l'université libanaise. À partir des 
questionnaires administrés auprès d'un échantillon de 65 enseignants stagiaires, cette étude de cas explore le concept d'apprentissage hybride de leurs points de vue. Le questionnaire comprenait cinq axes principaux basées sur la littérature tels (a) le processus d'apprentissage, (b) le soutien du formateur, (c) le matériel, (d) la collaboration et (e) la technologie. Les résultats ont indiqué que les enseignants étaient satisfaits à la fois de leur participation au cours et des connaissances acquises lors de la formation. Les résultats ont également révélé un certain nombre de facteurs à prendre en considération lors de la conception de ce genre de cours dans le contexte libanais.

Mots-clés: formation hybride; Liban; enseignement universitaire; technologie éducation, formation continue, enseignants.

\section{Introduction}

Le cadre pour l'apprentissage dans le $21^{\text {ème }}$ siècle a été développé pour répondre aux compétences essentielles qu'un apprenant doit démontrer pour être un citoyen efficace du monde. Les compétences telles que les 4C: la pensée critique, la communication, la collaboration et la créativité, ainsi que les compétences en alphabétisation telles que l'alphabétisation de l'information, l'éducation aux médias et l'alphabétisation technologique, sont alignées avec les normes de base et sont disponibles pour les enseignants qui souhaitent intégrer telles compétences dans leurs cours (Magner, Soulé, et Wesolowski, 2011). Même si ces cadres définissent et favorisent les compétences et l'importance de l'alphabétisation technologique, beaucoup d'instructeurs ont toujours du mal à mettre en œuvre efficacement la technologie dans le cadre de leurs pratiques pédagogiques. Pour que la technologie aide efficacement l'apprentissage des étudiants, les aspects pédagogiques, l'utilisation des applications, l'attitude des apprenants et des enseignants envers la technologie doivent être soigneusement prises en compte.

La qualité de la façon dont la technologie est abordée dans les programmes de formation des enseignants est l'une des conditions qui détermine comment les étudiants utilisent la technologie dans leurs classes (Tondeur, van Braak, Sang, Voogt, Fisser et Ottenbreit-Leftwich, 2012). Dans les programmes de formation des enseignants, la technologie reçoit peu d'attention, que ce soit la manière dont elle peut être utilisée dans l'enseignement ou la pédagogie de cette formation (Chien, Chang, Yeh et Chang, 2012). Il semble qu'un petit nombre d'enseignants puissent utiliser la technologie de manière diversifiée et flexible pour créer un apprentissage axé sur l'élève (Bang et Luft, 2013; Gao, Wong, Choy et Wu, 2011). Un exemple d'utilisation de la technologie de manière flexible pour créer un apprentissage plus axé sur l'élève en classe est le principe des classes inversées («Flipping 
the Classroom», Davis et al., 2013), qui se réfère à un modèle pédagogique qui «ajuste le design et la prestation des instructions afin que les élèves prennent l'initiative et la responsabilité d'apprendre avant la classe et que l'instructeur puisse passer le temps de classe à travailler sur les activités d'apprentissage appliquées (Davis et al., 2013, p. 241).

Ce manque d'attention à la technologie dans la formation des enseignants signifie que la plupart des formations pour intégrer la technologie dans l'enseignement se font au cours de la pratique scolaire, après que les enseignants sont entrés dans la profession. Cependant, l'attention portée à la technologie dans les programmes de préparation des enseignants ne suffit pas; C'est le «comment» ce qui compte. Finger et al. (2013) et Sweeney et Drummond (2013) ont conclu que la formation initiale ou continue des enseignants ne devrait pas seulement se concentrer sur la façon d'utiliser la technologie, mais également sur la façon dont la technologie s'intègre avec la connaissance pédagogique et du contenu. Les cours de technologie se révèlent inefficaces pour fournir aux candidats durant la formation des enseignants une préparation appropriée pour intégrer efficacement la technologie dans leurs instructions, bien que ces cours continuent à être une partie sérieuse de nombreux programmes de formation des enseignants (Gronseth et al., 2010).

\section{Cadre théorique}

\subsection{Approches technologiques dans la formation des enseignants}

Le passage d'une approche basée sur des cours de technologie isolés à une approche où la technologie est intégrée dans tous les cours (Tondeur, et al., 2012) s'harmonise avec le cadre éducatif connu sous le nom de TPACK (Mishra \& Koehler, 2006). Le cadre de TPACK a ses racines dans le travail de Shulman (1986) qui a suggéré que le bon enseignement implique un mélange de contenu et de connaissances pédagogiques. Ainsi, Mishra et Koehler (2006) ont suggéré que l'intégration de la technologie exige que les enseignants possèdent non seulement un contenu solide, des connaissances pédagogiques et technologiques, mais aussi la formation doit assurer une cohérence entre les connaissances de base. En raison de la nature interactive de ces trois bases de connaissances, on pourrait remettre en question l'efficacité des cours de technologie purs pour l'intégration de la technologie dans les classes.

Wetzel, Buss, Foulger et Lindsey (2014) ont évalué la mise en œuvre d'un programme pour intégrer la technologie dans les cours dans le cadre d'un programme de formation des enseignants. Ils ont mis en place des groupes de discussion pour les enseignants candidats pour révéler les succès et les dilemmes de la formation. Leur principale recommandation - plus de possibilités pour les instructeurs de développer leur expertise pour modéliser une approche basée sur la technologie et pour fournir plus d'apprentissage pratique aux enseignants ; de même, fournir aux enseignants candidats plus de 
possibilités d'appliquer ce qu'ils ont appris en classe et de mettre en œuvre une approche basée sur la technologie dans leur propre enseignement.

\subsection{Contexte libanais}

Les technologies de l'information et de la communication (TIC) sont devenues, ces dernières années, l'un des éléments de base de la société libanaise. La popularité des TIC parmi les citoyens libanais croît à un rythme exponentiel, et est devenue un outil indispensable dans l'accomplissement de leurs tâches quotidiennes.

Cette étude vient d'une conscience profonde de l'importance de l'enseignant dans la construction d'une société moderne qui aspire constamment à l'innovation. Ceci peut avoir lieu par l'amélioration des méthodologies et des outils pour assurer un meilleur investissement des potentiels des apprenants; ce qui témoigne positivement le développement économique et social et culturel du pays.

Les programmes de préparation des enseignants et leur développement professionnel révèlent la réalité de l'apprentissage, l'éducation et la perception réelle du rôle de l'école; ceci aidera nécessairement à développer de nouvelles politiques et programmes fondés sur une compréhension objective de la réalité de l'éducation dans le pays ainsi que sur le développement d'un plan économique prometteur qui prend en compte les variables cognitives et la technologie dans la société actuelle et leur impact sur tout aspect de la société et de la vie, y compris l'éducation et le rôle de l'enseignant en premier lieu.

Une étude réalisée par la Banque Mondiale en 2010 (Saber country report 2010) ${ }^{1}$ a montré que la formation des enseignants au Liban est au niveau « émergeant» par rapport à leurs collègues dans les pays développées. Ceci peut être au niveau des exigences du métier, formation initiale ou continue, ou au niveau des capacités des enseignants.

Dans le contexte de l'ère numérique et des programmes d'études pour développer les compétences du 21ème siècle, la technologie est considérée comme essentielle pour fournir des environnements d'apprentissage actuels et favorables et concurrencer sur le marché mondial. Alors que le programme national libanais ne s'y attaque pas encore, certaines écoles privées locales, intègrent la technologie dans leurs salles de classe depuis des années. À cette fin, le Ministère de l'Education et de l'Enseignement Supérieur (MEES) a publié un plan national de technologie éducative en $2012^{2}$. Le but du plan était que tous les étudiants aient accès à la technologie en classe pour soutenir les objectifs curriculaires en 2017. Le fondement du plan est principalement une revue de la littérature avec des preuves pratiques, empiriques locales mais

\footnotetext{
${ }^{1}$ http://wbgfiles.worldbank.org/documents/hdn/ed/saber/supporting_doc/CountryReports/TC H/SABER_Teachers_Lebanon_CR_Final_2010.pdf

${ }^{2}$ http://www.mehe.gov.lb/Uploads/file/TLSP.pdf
} 
limitées sur l'intégration des technologies de l'information et de la communication (TIC). Le plan discute comment les perceptions sur les technologies de l'information et de la communication (TIC) dans les écoles ont changé au cours des vingt-cinq dernières années dans le monde, parallèlement au développement de la technologie. Le plan discute comment était la situation au Liban lorsque les ordinateurs ont été introduits pour la première fois dans les écoles ; l'accent était mis sur l'enseignement et l'apprentissage «des TIC» et la technologie, étant nouvelle et relativement limitée, imposait que les étudiants sachent comment l'utiliser et le souci était principalement de les préparer à un avenir dans lequel les ordinateurs joueraient un rôle important. Mais avec le développement rapide et la complexité croissante et la gamme des utilisations des TIC ainsi que le développement de leurs liens de communication à travers l'Internet, ces outils sont devenus très puissants tel que maintenant l'accent est sur l'enseignement et l'apprentissage «avec les TIC». En conséquence, les TIC sont une question stratégique pour l'école qui nécessite une gestion prudente de la part d'un groupe dirigeant. Il est crucial de reconnaître que les TIC peuvent changer fondamentalement la façon dont les élèves apprennent; les autres utilisations principales des TIC, pour l'administration et pour la communication, sont également des considérations pour les directeurs d'école, mais l'accent doit être mis sur l'apprentissage.

\subsection{Contributions des organisations internationales au développement des TICE au Liban}

En raison du succès de l'initiative TICE dans les écoles britanniques, le British Council a décidé de lancer deux projets dans cinq pays du ProcheOrient et de l'Afrique du Nord y compris le Liban. Au Liban, ces projets ont été lancés en tant que joint-ventures entre le British Council et le Centre de Recherche et de Développement Pédagogique (CRDP) en 2005. Le premier projet concerne les TIC pour les enseignants d'anglais, de sciences et de mathématiques au Liban, et le second est le Leadership stratégique en TIC (SLICT) pour les directeurs d'école. Une communauté de pratique en ligne régionale a été créée pour soutenir les enseignants participants, ce qui constituait un élément clé de l'aspect de suivi du programme de formation des enseignants.

L'enseignement de qualité pour l'accès et l'amélioration de l'éducation de base financé par l'USAID (QITABI, septembre 2014-septembre 2018) visait à élargir l'accès équitable et améliorer les résultats d'apprentissage pour les élèves vulnérables dans les écoles publiques libanaises. Le projet QITABI est mis en œuvre par quatre partenaires: World Learning, Ana Aqra', AMIDEAST et Management Systems International (MSI). Ces partenaires apportent une assistance technique et un renforcement des capacités dans le cadre de trois 
composantes du projet (résultats intermédiaires dans la stratégie de coopération au développement de la mission de l'USAID):

Composante 1: Améliorer les résultats en lecture pour les élèves des écoles primaires;

Composante 2: élargir l'accès à une éducation sûre et pertinente pour les élèves des écoles publiques vulnérables;

Composante 3: Renforcement de la gestion (résilience) dans le système éducatif pour mieux diriger et suivre l'éducation.

La Banque Mondiale a réalisé en 2015 «Tech For Kids», une série d'ateliers destinés à familiariser les enfants, les enseignants et les membres de l'administration libanaise avec l'utilisation d'outils de technologies de l'information et de la communication innovants qui favorisent la résolution de problèmes et la collaboration en classe, ainsi qu'à les initier à de nouvelles méthodes qui stimulent la créativité.

\subsection{Formations technologiques : plusieurs voies}

Les institutions éducatives au Liban ont développé leurs approches de l'intégration de la technologie dans l'éducation en plusieurs étapes selon différentes perspectives pour chaque étape, mais ce développement n'était pas le résultat d'un processus de maturité naturelle envers ce sujet. Par exemple, entre 2004 et 2008, les approches d'intégration ont été fortement influencées par les produits Microsoft, les programmes et le réseau PIL, conçu pour former les enseignants selon cette vision.

Entre 2009 et 2014, le concept d'intégration de la technologie dans l'éducation s'est limité au-fonctionnement du logiciel Activinspire: toute la formation des enseignants s'est concentrée sur ce programme et les directions scolaires ont obligé les enseignants à préparer des cours électroniques sur ce programme. À ce stade, les enseignants étaient supposés être des concepteurs et des auteurs, avec une formation technique très modeste, sans aucune expérience ou vision pédagogique.

Entre 2012 et 2016, une nouvelle voie s'est ouverte, celle de l'apprentissage mobile, basée sur une armoire contenant des tablettes et un système de gestion de l'apprentissage (LMS), a été conçue. Une fois de plus, l'enseignant devait s'adapter à passer de l'apprentissage collectif à l'apprentissage différencié, et les technologues ont avancé le BYOD «bring your own device ». Ici l'incident a été catastrophique pour l'enseignant, car il a été placé brusquement, sans formation préalable ni accompagnement de la part de la direction devant un mode d'apprentissage mixte très avancé.

À partir de 2013, le «robot éducatif» a donné naissance au STEM et STEAM (intégration des mathématiques, de la technologie, de l'ingénierie et de la science dans un projet). Bien sûr, l'idée est merveilleuse et idéale du point de vue éducatif, mais hors contexte elle perd toutes ses caractéristiques. 
Ces technologies variées, non prévues dans les formations ni dans les pratiques antérieures, n'ont pas facilité la modification des pratiques éducatives. L'intégration de la technologie dans l'éducation doit être une combinaison de plusieurs éléments pour réussir. Toutes les tentatives précédentes ont été influencées par la pression médiatique et marketing des entreprises technologiques, de sorte qu'elles vont investir sans remplir les conditions de succès et en premier les qualifications des enseignants et leur mission pédagogique.

Malheureusement, la plupart des instituts éducatifs, et dans leur hâte de rattraper et d'introduire de nouveaux outils numériques, ne font souvent que déployer des technologies éducatives dans les salles de classe sans modifier l'éducation elle-même pour l'adapter au futur (MEHE, 2012), tandis que les enseignants n'ont pas du mal à savoir s'ils utilisent la technologie ou non, mais plutôt comment. Dans l'étude en cours, un cours de formation des enseignants basé sur une approche hybride (blended learning) a été évalué afin de mieux comprendre la valeur de cette approche pour intégrer la technologie dans l'enseignement.

La définition de l'apprentissage mixte fournie par Williams (2002) comme la combinaison de l'apprentissage en face - à - face et en ligne a été contestée par certains chercheurs comme insuffisante, car elle implique de «greffer» la technologie sur un cours traditionnel comme contenu additionnel ou supplémentaire et ignore le besoin de repenser la conception ou la pédagogie du cours (Vaughan, 2007). La prestation de cours d'apprentissage hybride implique le déplacement d'une partie importante des activités d'apprentissage en ligne. Un mélange de méthodes traditionnelles (enseignement en classe en face-à-face), d'enseignement basé sur la technologie, y compris la communication en ligne, les activités et la prestation, entraîne des changements importants dans la prestation du cours; par exemple, raccourcir le «temps du présentiel» (Garrison, Kanuka et Howes 2002). Ces cours se distinguent des cours d'apprentissage à distance offerts en ligne, comme le soulignent Dziuban, Hartman et Moskal (2004) à cause d'un remaniement de la prestation et de la pédagogie (Vaughan, 2007).

\section{Contexte de l'étude : Descriptif du cours}

En prenant en considération les arguments ci-dessus, la définition de l'apprentissage hybride comme «intégration significative des activités de classe en ligne et traditionnelles en face - à - face» (Alebaikan et Troudi 2010, p. 50) est suffisante pour la portée de cet article. Cette recherche vise à étudier les perceptions, et les pratiques liées à l'apprentissage hybride dans un cours de formation au niveau « Diplôme Professionnel Universitaire », au « Centre des 
Sciences du Langage et de la Communication $»^{3}$, conçu pour améliorer les compétences des enseignants en service vis-à-vis de l'intégration des TICE dans leurs cours et à identifier les facteurs qui facilitent ou entravent l'adoption et la mise en œuvre de l'apprentissage mixte l'Université Libanaise en général.

Les étudiants ont l'opportunité d'expérimenter de la théorie et des meilleures pratiques dans les domaines de l'éducation et de la formation exploré des compétences de conception pédagogique et des connaissances associées aux méthodologies d'identification des problèmes, à l'analyse des apprenants, à l'analyse des tâches, aux objectifs pédagogiques, aux stratégies d'enseignement, aux messages pédagogiques et à l'évaluation.

Easyclass est le système de gestion de l'apprentissage $\left(L M S^{4}\right)$ adopté pour créer une classe virtuelle pour stocker le matériel en ligne, gérer les discussions, donner des devoirs, des quiz et fournir aux élèves des commentaires en un seul endroit. Edmodo, un autre site éducatif a été utilisé pour promouvoir la communication et échanger les idées, et les conseils utiles.

L'apprentissage mixte dans le cours était une combinaison de modalités pédagogiques (sur site: Easyclass, apprentissage à l'auto-apprentissage, Edmodo), de supports de diffusion (documents sur Internet, présentations PowerPoint); des méthodes pédagogiques (sessions en face-à-face ou basées sur la technologie) et des technologies basées sur le Web (forums de discussion, blogs). La manière dont la combinaison est effectuée dépend de critères tels que les objectifs d'apprentissage, le contenu du cours, l'expérience de l'enseignant et le style d'enseignement, les caractéristiques des apprenants, etc. (Dziuban, Hartman \& Moskal, 2005).

Le modèle adopté dans le cours était celui de Barnum et Paarmann (2002) qui ont présenté quatre stratégies à inclure dans un modèle d'apprentissage mixte. Ces stratégies sont les suivantes:

- la diffusion sur le Web, qui favorise l'indépendance et l'autonomie dans l'apprentissage;

- le face-à-face, dans lequel l'interaction humaine est nécessaire pour construire une compréhension plus profonde;

- la création de livrables, s'attendant à ce que les apprenants créent des produits (taches hebdomadaires);

- et l'extension collaborative de l'apprentissage - les groupes se rencontrent une fois par semaine pour partager et approfondir.

\footnotetext{
${ }^{3}$ Une des branches de la Faculté des Lettres et Sciences Humaines à L’Université Libanaise, qui ouvre la technologie aux sciences humaines et, plus spécifiquement, à la linguistique.

${ }^{4}$ LMS: Learning Management System, un système qui gère l'apprentissage.
} 


\section{Problématique}

Enseigner aujourd'hui nécessite des enseignants compétents, en particulier en utilisant des ordinateurs et d'autres outils technologiques. Malheureusement, les enseignants indiquent toujours qu'ils hésitaient à utiliser la technologie et ne se sentaient pas préparés et craignaient de l'intégrer dans leur enseignement en classe. Leur réticence à utiliser la technologie est principalement due à leurs opinions négatives vis-à-vis de la présence de la technologie dans le cadre de leurs nouvelles méthodes d'enseignement.

D'autre part, le ministère de L'éducation et de l'enseignement supérieur au Liban (MEES), suite à des exigences internationales de contrôle de qualité, exige que l'enseignement soit de plus en plus assiste par ordinateur dans les écoles privées et publiques. Par suite, il est important d'évaluer les points de vue des enseignants sur la relation entre technologie et enseignement ainsi que sur leurs compétences technologiques ainsi que sur l'impact potentiel des TICE afin de déterminer le succès de toute initiative dans ce domaine. De même, puisque les pratiques des enseignants dans leurs classes dépendent de leurs représentations et de leurs connaissances, cette recherche a été menée lors d'une formation hybride des enseignants afin de savoir leurs perspectives sur la formation qu'ils ont suivis car ceci peut influencer leurs activités de classe.

Dans cette étude, le cours visé, "Conception de l'Apprentissage en Ligne », commence par l'analyse des besoins des destinataires du cours. Il présente, de façon simple, les fondamentaux permettant la structuration et la scénarisation d'un cours en ligne (spécifications d'entrée, principaux acteurs, intentions pédagogiques, modèles d'apprentissage, activités de différents types). Il explique également les principes généraux de la mise en œuvre d'un cours suite à la mise en ligne de ce dernier ainsi que son évaluation.

En explorant le point de vue des enseignants sur l'apprentissage mixte, cette étude de cas peut informer les personnes qui gèrent, planifient et prennent des initiatives en matière de perfectionnement professionnel, y compris la formation mixte et peut servir comme étude pilote pour les formations ultérieures.

Les questions de recherche suivantes ont été formulées:

(1) Quelles sont, d'après les enseignants, leurs compétences et leurs pratiques par rapport aux TICE avant et après la formation?

(2) Quels sont, d'après les enseignants, les facteurs qui influencent l'utilisation de la technologie en classe ?

(3) Comment le cours de formation hybride a- t-il été évalué par les enseignants du point de vue contenu, communication et méthodes?

\section{Méthodologie}

Cette recherche est une étude de cas réalisée pour repérer les points de vue des enseignants sur la technologie et l'enseignement, ainsi que leurs 
compétences perçues en matière TICE suite à une formation hybride. Les répondants à l'enquête menée sont des enseignants d'écoles privées reparties sur 4 gouvernorats au Liban et ils ont tous assisté à ce cours. Un total de 65 enseignants a été sélectionné comme échantillon pour cette étude.

\subsection{Instrument}

Un questionnaire a été distribué aux enseignants. Le questionnaire est divisé en quatre sections. L'information démographique des enseignants (section 1) a été développée pour déterminer les antécédents des enseignants et elle constitue la première section. La deuxième section (section 2 ) comporte une série de questions visant à obtenir des renseignements sur l'accès disponible aux équipements technologiques. Quant à la troisième section elle s'intéresse à l'échelle de compétences informatiques (section 3) et elle a été utilisée pour mesurer la compétence perçue des enseignants vis-à-vis des TICE à l'aide d'une échelle de Likert de 1 à 5.

Dans la dernière section (section 4), les répondants devraient exprimait leur accord ou désaccord concernant le niveau de satisfaction sur tous les aspects du cours.

\subsection{Validité}

Les éléments des sections 2 et 3 de l'instrument ont été vérifiés quant à leur validité par trois experts de différents domaines tel l'informatique et la pédagogie. Les experts ont évalué et reconstruit le contenu des instruments et plusieurs modifications ont été apportées. Un test pilote a été effectué pour obtenir la fiabilité des instruments modifiés. Il a été administré par le chercheur à 10 enseignants. Les Cronbach alpha rapportés pour les opinions sur la technologie et les échelles de compétence en informatique du questionnaire modifié étaient supérieure à 0,6 (Tableau 1). Les scores de fiabilité ci-dessous fournissent la preuve que les mesures sont cohérentes.

\begin{tabular}{|c|c|c|}
\hline & N of Items & Cronbach Alpha \\
\hline Niveau de maîtrise & 11 & 0.864 \\
\hline Activités & 10 & 0.920 \\
\hline Rechercher de l'information & 5 & 0.745 \\
\hline Diffuser de l'information & 6 & 0.779 \\
\hline Après cours & 9 & 0.892 \\
\hline Facteurs négative & 6 & 0.632 \\
\hline Capabilité & 7 & 0.963 \\
\hline Tableau 1 : valeurs de Cronbach Alpha pour les sections du questionnaire \\
\hline
\end{tabular}

\section{Résultats}

Les données collectées ont été analysées à l'aide du progiciel statistique pour les sciences sociales (SPSS), à l'aide de statistiques descriptives et inférentielles. La fréquence, le pourcentage, la moyenne et l'écart type ont été calculés dans le but d'analyser les variables. 


\subsection{Caractéristiques de l'échantillon}

$8.7 \%$ de la population était des hommes et $88.4 \%$ des femmes. La majorité d'entre eux avait entre 31 et 40 ans et avaient moins que 10 ans d'expérience comme enseignant.

Les enseignants travaillaient dans tous les cycles élémentaires, complémentaires et secondaires. $91,3 \%$ ont accès ordinateur à domicile et $81,2 \%$ ont accès internet.

\subsection{Les enseignants au Travail}

\subsubsection{Valeur accordée à la technologie comme outil d'enseignement- apprentissage}

\begin{tabular}{|c|c|c|}
\hline & Fréquence & Pourcentage \\
\hline Très précieux & 33 & $47.8 \%$ \\
\hline Précieux & 30 & $43.5 \%$ \\
\hline Ni valable ni non valable & 4 & $5.8 \%$ \\
\hline Modérément précieux & 2 & $2.9 \%$ \\
\hline Total & 69 & $100.0 \%$ \\
\hline
\end{tabular}

Le tableau ci-dessus montre que 91,3\% (47,8\% + 43,5\%) des enseignants valorisent l'utilisation de la technologie en tant qu'outil éducatif, alors que $2,9 \%$ ne lui donnaient pas d'importance.

\subsubsection{Les ressources à la disposition des enseignants au travail}

Environ $100 \%$ ont déclaré utiliser l'ordinateur portable, $96 \%$ ont un téléphone smart, $84 \%$ utilisent Internet, $79 \%$ un ordinateur de bureau et $79 \%$ un graveur de CD / DVD. Le pourcentage d'utilisation des tableaux interactifs était de $71 \%$, contre $69 \%$ pour l'iPad et $57 \%$ pour l'appareil photo numérique, $57 \%$ pour un appareil photo, $57 \%$ pour le scanner, et $11,76 \%$ pour le caméscope, qui est le plus bas.

\begin{tabular}{|c|c|}
\hline & Pourcentage \\
\hline Ordinateur portable & 100 \\
\hline Téléphone smart & 95.58824 \\
\hline Accès Internet & 83.82353 \\
\hline Ordinateur de bureau & 79.41176 \\
\hline Graveur CD / DVD & 79.41176 \\
\hline Tableau blanc interactif & 70.58824 \\
\hline Tablette / iPad & 69.11765 \\
\hline Scanner & 57.35294 \\
\hline Appareil photo & 57.35294 \\
\hline Tablette & 52.94118 \\
\hline Appareil photo numérique & 47.05882 \\
\hline Caméra vidéo (ou Flip) & 11.76471 \\
\hline Tableau 3 : ressources disponibles au travail
\end{tabular}


6.2.3. Fréquence de l'Utilisation de la technologie comme un outil d'enseignement dans la salle de classe

\section{Figure 1: Fréquence d'utilisation}

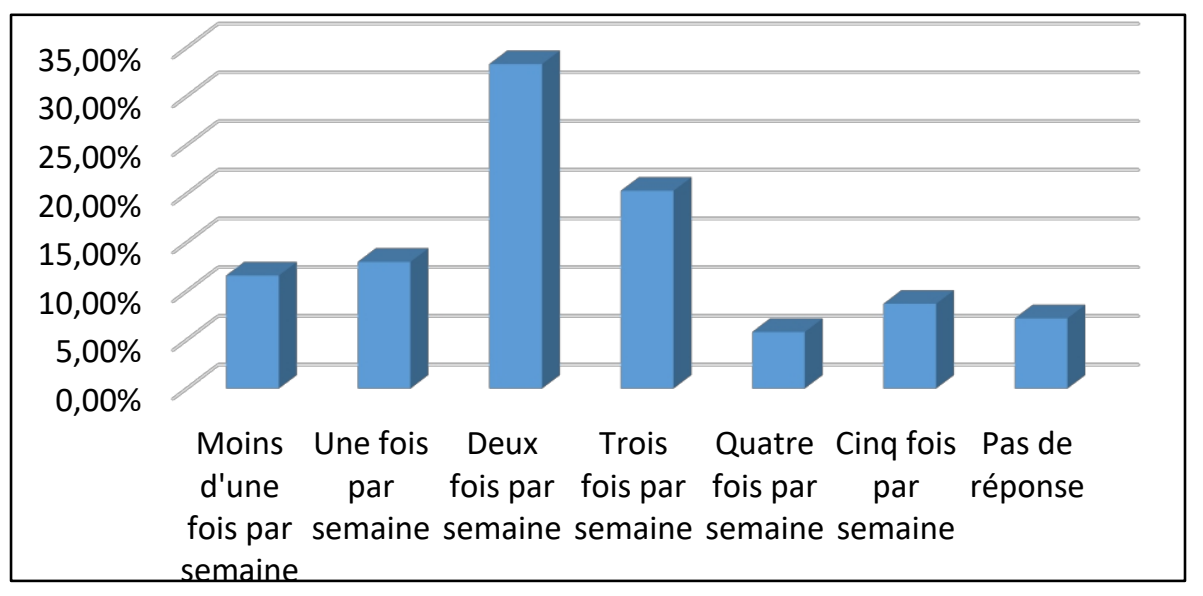

$33,3 \%$ des enseignants utilisent la technologie comme un outil pédagogique deux fois par semaine, tandis que 20,3\% l'utilisent trois fois par semaine.

\subsubsection{Capacités technologiques déjà acquises}

\begin{tabular}{|c|c|c|c|c|c|c|}
\hline Niveau de maîtrise & Jamais & $\begin{array}{c}\text { Presque } \\
\text { jamais }\end{array}$ & $\begin{array}{c}\text { De temps } \\
\text { en temps }\end{array}$ & $\begin{array}{c}\text { Assez } \\
\text { souvent }\end{array}$ & $\begin{array}{c}\text { Très } \\
\text { souvent }\end{array}$ & $\begin{array}{c}\text { Pas de } \\
\text { réponse }\end{array}$ \\
\hline $\begin{array}{c}\text { Traitement de texte (Microsoft } \\
\text { Word, etc.) }\end{array}$ & $0.0 \%$ & $1.4 \%$ & $13.0 \%$ & $31.9 \%$ & $49.3 \%$ & $4.3 \%$ \\
\hline $\begin{array}{c}\text { Logiciels de présentation } \\
\text { (Microsoft PowerPoint, etc.) }\end{array}$ & $2.9 \%$ & $1.4 \%$ & $23.2 \%$ & $30.4 \%$ & $37.7 \%$ & $4.3 \%$ \\
\hline Tableur (Microsoft Excel, etc.) & $7.2 \%$ & $15.9 \%$ & $29.0 \%$ & $30.4 \%$ & $13.0 \%$ & $4.3 \%$ \\
\hline $\begin{array}{c}\text { Éditeur de pages Web (Microsoft } \\
\text { FrontPage, Dreamweaver, etc.) }\end{array}$ & $47.8 \%$ & $17.4 \%$ & $13.0 \%$ & $8.7 \%$ & $4.3 \%$ & $8.7 \%$ \\
\hline $\begin{array}{c}\text { Éditeur d'images (Adobe } \\
\text { Photoshop, Paint, Corel Draw, } \\
\text { etc.) }\end{array}$ & $17.4 \%$ & $17.4 \%$ & $26.1 \%$ & $20.3 \%$ & $13.0 \%$ & $5.8 \%$ \\
\hline $\begin{array}{c}\text { Courrier électronique } \\
\text { Moteurs de recherche (Google, } \\
\text { AltaVista, etc.) }\end{array}$ & $13.0 \%$ & $10.1 \%$ & $5.8 \%$ & $30.4 \%$ & $34.8 \%$ & $5.8 \%$ \\
\hline $\begin{array}{c}\text { Outils de communication } \\
\text { (clavardage ou « chat », forums) }\end{array}$ & $14.5 \%$ & $14.5 \%$ & $17.4 \%$ & $24.6 \%$ & $37.7 \%$ & $4.3 \%$ \\
\hline $\begin{array}{c}\text { Appareil photo numérique, } \\
\text { Caméra Web (« Webcam ») }\end{array}$ & $26.1 \%$ & $20.3 \%$ & $14.5 \%$ & $18.8 \%$ & $15.9 \%$ & $4.3 \%$ \\
\hline Tableau 4 : capacités technologiques des enseignants & & & $7.2 \%$ \\
\hline
\end{tabular}


Cette question était relative aux capacités technologiques que les enseignants possédaient avant leur formation à l'université. Les résultats ont révélé que $(31,9+49,3 \%+13)=94,2 \%$ ont déclaré pouvoir utiliser le programme Word avec des fréquences différentes, 23,2 + 30,4 + 37,7\% (90,9\%) logiciels de présentation tel le PowerPoint.

Pour la communication, $(17,4+24,6+37,7 \%)=79,7 \%$ pour Moteurs de recherche (Google, AltaVista, $15,9+24,6+20,3 \%$ ) $=60,8 \%$ clavardage chat», forums)

Les autres logiciels sont utilisés moins fréquemment: $(26,120,3+$ $13 \%)=59,4 \%$ pour éditeur d'images (Photoshop...), $(23,2+30,4+23 \%)=$ $56,6 \%$ pour Excel, $(13+8,7+4,3 \%)=26 \%$ Éditeur de pages Web, et $(14,5+$ $18,8+4,3 \%)=39 \%$ utilisaient le Webcam.

Les résultats de l'analyse de l'alphabétisation numérique ont montré que la plus grande proportion d'enseignants de l'échantillon utilisait Word et PowerPoint bien avant leur formation à l'université.

\subsubsection{Les buts de l'utilisation de la technologie en classes}

La fréquence avec laquelle les technologies sont intégrées aux activités d'apprentissage

\begin{tabular}{|c|c|c|c|c|c|c|}
\hline Activités & Jamais & $\begin{array}{l}\text { Presque } \\
\text { jamais }\end{array}$ & $\begin{array}{l}\text { De temps } \\
\text { en temps }\end{array}$ & $\begin{array}{c}\text { Assez } \\
\text { souvent }\end{array}$ & $\begin{array}{c}\text { Très } \\
\text { souvent }\end{array}$ & $\begin{array}{l}\text { Pas de } \\
\text { réponse }\end{array}$ \\
\hline $\begin{array}{c}\text { Enseignement (ex.: exercices de } \\
\text { répétition, pratique, travaux dirigés, } \\
\text { rattrapage) }\end{array}$ & $1.4 \%$ & $7.2 \%$ & $18.8 \%$ & $40.6 \%$ & $23.2 \%$ & $8.7 \%$ \\
\hline $\begin{array}{l}\text { Communication (ex. : courriel, ICQ, } \\
\text { conférences assistées par ordinateur) }\end{array}$ & $5.8 \%$ & $10.1 \%$ & $26.1 \%$ & $30.4 \%$ & $17.4 \%$ & $10.1 \%$ \\
\hline $\begin{array}{l}\text { Organisation (ex. : banque de } \\
\text { données, tableurs, rédaction de } \\
\text { rapports, planification) }\end{array}$ & $8.7 \%$ & $13.0 \%$ & $21.7 \%$ & $23.2 \%$ & $21.7 \%$ & $11.6 \%$ \\
\hline $\begin{array}{c}\text { Analyse/programmation (ex.: } \\
\text { statistiques, tableaux, graphiques, } \\
\text { dessins, robotique) }\end{array}$ & $10.1 \%$ & $13.0 \%$ & $31.9 \%$ & $20.3 \%$ & $14.5 \%$ & $10.1 \%$ \\
\hline $\begin{array}{c}\text { Détente (ex. : jeu, films, vidéos, } \\
\text { musique) }\end{array}$ & $11.6 \%$ & $11.6 \%$ & $26.1 \%$ & $21.7 \%$ & $20.3 \%$ & $8.7 \%$ \\
\hline $\begin{array}{l}\text { Exploration (ex. : simulations, } \\
\text { expériences, situations de découverte, } \\
\text { remue-méninges) }\end{array}$ & $2.9 \%$ & $14.5 \%$ & $27.5 \%$ & $24.6 \%$ & $15.9 \%$ & $14.5 \%$ \\
\hline $\begin{array}{c}\text { Créativité (ex. : publication assistée } \\
\text { par ordinateur, vidéos numériques, } \\
\text { caméras numériques, scanners, } \\
\text { graphiques) }\end{array}$ & $10.1 \%$ & $11.6 \%$ & $29.0 \%$ & $18.8 \%$ & $17.4 \%$ & $13.0 \%$ \\
\hline $\begin{array}{l}\text { Expression (ex. : traitement de texte, } \\
\text { journaux en ligne) }\end{array}$ & $11.6 \%$ & $30.4 \%$ & $13.0 \%$ & $18.8 \%$ & $11.6 \%$ & $14.5 \%$ \\
\hline $\begin{array}{c}\text { Evaluation (ex. : devoirs, dossiers, } \\
\text { tests) }\end{array}$ & $13.0 \%$ & $13.0 \%$ & $20.3 \%$ & $14.5 \%$ & $23.2 \%$ & $15.9 \%$ \\
\hline $\begin{array}{c}\text { Renseignements (ex. : Internet, CD- } \\
\text { ROM) }\end{array}$ & $5.8 \%$ & $5.8 \%$ & $13.0 \%$ & $21.7 \%$ & $39.1 \%$ & $14.5 \%$ \\
\hline \multicolumn{7}{|c|}{ Tableau 5 : utilisations des technologies en classe } \\
\hline
\end{tabular}


$(18,8+40,6+8,7 \%)=82,6 \%$ des enseignants utilisent la technologie pour les activités d'enseignement (ex:. Exercices de répétitions, travaux pratiques, travaux dirigés), \%73.8 (\%39.1+\%21.7+\%13.0) l'utilisent pour avoir des informations (ex. : Internet, CD-ROM)

Alors que $(26,1+30,4+17,4 \%)=73,9 \%$ l'utilisent pour la communication (ex .: Courriel, web conférences), $(21,7+23,2+21,7 \%)=$ $66,7 \%$ l'utilisent pour l'organisation de l'information (tableurs, Rapports). Le même pourcentage est signalé pour les statistiques, tableaux, Graphiques).

D'autres utilisations ont été citées tels que : $(26,1 \%+21,7 \%+20,3 \%$ )$=68,1 \%$ pour la détente (ex .: Jeu, films, vidéos, musique), le même pourcentage pour la découverte, (exploration (ex .: simulations, expériences, situations de découverte, Remue-méninges)). Par rapport à (29,0\% 18,8\% 17,4\%) 65,2\% pour produire des documents électroniques tels les vidéos Numériques, caméras Numériques, scanners, Graphiques)).

Peu d'enseignants l'utilisent pour l'évaluation.

\subsection{Perceptions vis-à-vis de la formation hybride}

\subsubsection{Capacités selon TPACK}

Le TPACK est considéré en tant que cadre permettant de comprendre et de décrire les types de connaissances nécessaires à un enseignant pour une intégration efficace des TIC. Ce qui importe n'est pas seulement les principales connaissances du cadre TPACK mais les interactions entre les différentes composantes. La présente recherche visait à obtenir les points de vue des enseignants après avoir suivi le cours concernant leur préparation technologique, pédagogique, technologique pédagogique, dans le but de développer des méthodes flexibles de réflexion et de réflexion sur les TIC, la conception et l'apprentissage qui aident au développement de TPACK.

\begin{tabular}{|c|c|c|c|c|c|c|}
\hline $\begin{array}{c}\text { Après avoir suivi ce cours, je me suis } \\
\text { senti pédagogiquement préparé car }\end{array}$ & Jamais & $\begin{array}{c}\text { Presqu } \\
\text { e } \\
\text { jamais }\end{array}$ & $\begin{array}{c}\text { De } \\
\text { temps } \\
\text { en } \\
\text { temps }\end{array}$ & $\begin{array}{c}\text { Assez } \\
\text { souve } \\
\text { nt }\end{array}$ & $\begin{array}{c}\text { Très } \\
\text { souve } \\
\text { nt }\end{array}$ & $\begin{array}{c}\text { Pas } \\
\text { de } \\
\text { répo } \\
\text { nse }\end{array}$ \\
\hline $\begin{array}{c}\text { J'ai reçu le soutien pédagogique } \\
\text { nécessaire pendant ce cours }\end{array}$ & $0.0 \%$ & $0.0 \%$ & $31.9 \%$ & $27.5 \%$ & $34.8 \%$ & $5.8 \%$ \\
\hline $\begin{array}{c}\text { J'ai eu une influence sur le contenu et les } \\
\text { activités du cours }\end{array}$ & $1.4 \%$ & $5.8 \%$ & $26.1 \%$ & $37.7 \%$ & $23.2 \%$ & $5.8 \%$ \\
\hline $\begin{array}{c}\text { Il y avait un bon équilibre entre les } \\
\text { activités en ligne et en classe }\end{array}$ & $0.0 \%$ & $4.3 \%$ & $24.6 \%$ & $46.4 \%$ & $21.7 \%$ & $2.9 \%$ \\
\hline $\begin{array}{c}\text { Je me sentais techniquement préparé pour } \\
\text { utiliser les différents TIC }\end{array}$ & $0.0 \%$ & $1.4 \%$ & $10.1 \%$ & $42.0 \%$ & $42.0 \%$ & $4.3 \%$ \\
\hline $\begin{array}{c}\text { Le format de l'apprentissage (mixte) n'a } \\
\text { pas rendu le cours exigeant }\end{array}$ & $1.4 \%$ & $11.6 \%$ & $34.8 \%$ & $23.2 \%$ & $20.3 \%$ & $8.7 \%$ \\
\hline $\begin{array}{c}\text { Mon style d'enseignement et } \\
\text { d'apprentissage correspond bien au } \\
\text { format du cours }\end{array}$ & $0.0 \%$ & $2.9 \%$ & $31.9 \%$ & $44.9 \%$ & $15.9 \%$ & $4.3 \%$ \\
\hline Les activités fournies sont convenables & $0.0 \%$ & $5.8 \%$ & $17.4 \%$ & $50.7 \%$ & $18.8 \%$ & $7.2 \%$ \\
\hline
\end{tabular}




\begin{tabular}{|c|c|c|c|c|c|c|}
$\begin{array}{c}\text { Je suis capable de concevoir, de } \\
\text { développer et d'évaluer des expériences } \\
\text { d'apprentissage et des évaluations } \\
\text { authentiques intégrant des outils et des } \\
\text { ressources technologiques contemporains } \\
\text { afin de maximiser l'apprentissage du } \\
\text { contenu }\end{array}$ & $0.0 \%$ & $4.3 \%$ & $20.3 \%$ & $47.8 \%$ & $24.6 \%$ & $2.9 \%$ \\
\hline $\begin{array}{c}\text { Je suis capable de faire preuve de } \\
\text { maîtrise des systèmes technologiques, } \\
\text { des compétences et des processus de } \\
\text { travail qui représentent un enseignant ou } \\
\text { un directeur dans une société mondiale et } \\
\text { numérique. }\end{array}$ & $0.0 \%$ & $5.8 \%$ & $30.4 \%$ & $39.1 \%$ & $20.3 \%$ & $4.3 \%$ \\
\hline
\end{tabular}

$(31,9 \%+27,5 \%+34,8 \%) 94,2 \%$ ont déclaré avoir reçu le soutien éducatif nécessaire pendant ce cours et \%87 (\%23.2+\%37.7+\%26.1) ont répondu qu'ils ont eu une influence sur le contenu et les activités du cours. $(24,6 \%+46,4 \%+21,7 \%) 92,7 \%$ ont indiqué qu'il y avait un bon équilibre entre les activités en ligne et en classe. $(42,0 \%+42,0 \%), 94,1 \%$ ont déclaré se sentir techniquement préparé pour utiliser les différents TIC, et $34,8 \%+23,2 \%$ $+20,3 \%=\% 90.3$ Le format de l'apprentissage (mixte également connu au Liban par le terme: blended learning) n'a pas rendu le cours exigeant, et (31,9\% $44,9 \% 15,9 \%)=92,7 \%$ considèrent que le format du cours correspond à leur style d'apprentissage.

$(17,4 \%+50,7 \%+18,8 \%) 86,9 \%$ des enseignants ont déclaré que les activités proposées étaient appropriées, $20,3 \%+47,8 \%+24,6 \%=92,7 \%$ ont estimé qu'ils étaient capables de concevoir et d'évaluer des expériences d'apprentissage et des évaluations authentiques intégrant des outils et des ressources technologiques contemporains afin de maximiser l'apprentissage du contenu, $(30,4 \%+39,1 \%+20,3 \%) 89,8 \%$ des enseignants ont déclaré être capables de maîtriser les systèmes technologiques, les compétences et les méthodes de travail qui représentent l'enseignant de la communauté mondiale et numérique.

\section{Capacités à l'issu de la formation}

Plus que $80 \%$ des enseignants ont noté une augmentation de leurs compétences technologiques suite à la formation :

$86.9 \%$ sont capables d'intégrer les TIC à vos stratégies pédagogiques

$80 \%$ sont capables de résoudre des problèmes techniques qui sont liés à l'utilisation des ordinateurs

$85.5 \%$ sont capables d'aider élèves à apprendre à utiliser les TIC

$85.5 \%$ sont capables d'aider les élèves lorsqu'ils font des recherches dans Internet 
\%86.9sont capables d'aider les élèves à réaliser des projets mettant à profit les TIC

\%86.9 sont capables d'amener les élèves à s'entraider pour résoudre des problèmes à l'aide des TIC.

82.6\% sont capables d'amener les élèves à exercer leur esprit critique face aux informations retrouvées dans Internet

\subsubsection{Les raisons pour lesquelles l'intégration de la technologie peut être limitée}

Cette partie a discuté les obstacles quant à l'intégration de la technologie en classe. La majorité des enseignants $(72,5 \%)$ a attribué ceci au manque d'équipements technologiques convenables ;

$62,3 \%$ attribuent ceci au manque de formation professionnelle en TICE, $42 \%$ pensent que l'intégration de la technologie prend beaucoup de temps aux dépens du contenu et $27.5 \%$ déclarent qu'ils sont incertains de l'effet de la technologie sur leurs pratiques en classe ainsi que sur la performance des élèves.

Les plus bas pourcentages 23\% ont été attribués aux formations continues en technologie que les enseignants trouvent assez différentes de leurs pratiques de classe ainsi qu'a la technologie elle-même que $21.7 \%$ pensent qu'elle n'est pas fiable.

\subsection{Opinions sur le Contenu du cours}

En ce qui concerne l'évaluation du contenu du cours par les enseignants et leur opinion, les réponses étaient les suivantes:

A propos du contenu théorique, $75,4 \%$ ont déclaré qu'il contribue à la maîtrise des connaissances de base et $88,4 \%$ ont dit qu'il était clair, et $79,7 \%$ considèrent qu'il est bien organisé, $52,2 \%$ ont remarqué que la quantité du contenu théorique était appropriée, et $85,5 \%$ ont vu une cohérence du contenu, cohérence entre l'apprentissage en «face - à - face » et l'apprentissage en ligne ; de même les enseignants ont trouvé que la façon dont le contenu est présenté est appropriée. Les résultats reflètent clairement la satisfaction des enseignants quant au contenu théorique et format du cours.

En ce qui concerne la partie pratique, $85,5 \%$ des enseignants a constaté qu'ils sont en mesure de mettre en place des activités et les inclure dans le cours, de même $85,5 \%$ ont été en mesure d'exploiter des activités variées pour guider les élèves, $81,2 \%$ ont trouvé l'approche hybride utile pour apprendre le contenu ainsi que la valeur de l'expérience vécue, 69,6\% trouvent que cette approche hybride exige une implication personnelle de l'élève pour l'appropriation du contenu, 52,2\% pensent que le temps nécessaire pour réaliser les activités était convenable avec la quantité du contenu et $82,6 \%$ considèrent que le cours comprenait un travail de coopération entre les élèves. 
En ce qui concerne l'interaction dans le cours offert en « face - à face » ou « en ligne » via le chat forum, 75,4\% des enseignants ont noté que le tuteur avait la connaissance du contenu pédagogique (PCK) nécessaire se rapportant aux méthodes d'enseignement appropriées aux contenus, 92,8\% ont répondu que le tuteur est toujours disponible pour aider les enseignants en ligne ou «face- à- face » et $88,4 \%$ ont déclaré que le tuteur contrôlait les apprentissages des élèves, donnait des feedbacks et les orientations nécessaires.

\section{Corrélations}

Pour connaître la nature de la relation entre les différents axes du questionnaire, le test de corrélation a été effectué pour considérer la relation entre les axes.

\begin{tabular}{|c|c|c|c|c|c|c|c|c|}
\hline \multicolumn{2}{|l|}{$N=69$} & $\begin{array}{c}\text { Niveau } \\
\text { de } \\
\text { maîtrise }\end{array}$ & Activités & $\begin{array}{c}\text { Rechercher } \\
\text { de } \\
\text { l'information }\end{array}$ & $\begin{array}{l}\text { Diffuser de } \\
\text { l'information }\end{array}$ & $\begin{array}{c}\text { Compétence } \\
\text { s acquises }\end{array}$ & Obstacles & $\begin{array}{c}\text { Capacit } \\
\text { é }\end{array}$ \\
\hline $\begin{array}{l}\text { Niveau de } \\
\text { maîtrise }\end{array}$ & Pearson & 1 & 0.536 & 0.421 & 0.470 & 0.357 & 0.159 & 0.340 \\
\hline \multirow{2}{*}{ Activités } & Pearson & 0.536 & 1 & 0.485 & 0.431 & 0.446 & 0.231 & 0.419 \\
\hline & p-value & 0.000 & & 0.000 & 0.000 & 0.000 & 0.056 & 0.000 \\
\hline \multirow{2}{*}{$\begin{array}{c}\text { Diffuser de } \\
\text { l'information }\end{array}$} & Pearson & 0.470 & 0.431 & 0.530 & 1 & 0.584 & 0.281 & 0.313 \\
\hline & p-value & 0.000 & 0.000 & 0.000 & & 0.000 & 0.019 & 0.009 \\
\hline \multirow{2}{*}{$\begin{array}{c}\begin{array}{c}\text { Compétences } \\
\text { acquises }\end{array} \\
\end{array}$} & Pearson & 0.357 & 0.446 & 0.504 & 0.584 & 1 & 0.196 & 0.547 \\
\hline & $\mathrm{p}$-value & 0.003 & 0.000 & 0.000 & 0.000 & & 0.106 & 0.000 \\
\hline
\end{tabular}

La valeur de $\mathrm{p}$ étant inférieure à $0,05(5 \%)$ indique une relation statistiquement significative entre les deux variables, et la valeur du coefficient Pearson indique la force de cette relation. Plus la valeur du coefficient Pearson est proche de 1, plus la relation est forte.

Par conséquent il y a une corrélation positive entre tous les axes de l'étude dont la valeur varie entre faible tel que $(0,281)$ et moyenne (tel que $0,536)$, à l'exception de l'axe « obstacles » où il n'y a pas de corrélation entre « Niveau de Maîtrise » et « compétences acquises » après formation.

Le tableau montre que les pratiques des stagiaires avant la formation sont directement reliées à leurs activités, la recherche et l'échange d'informations ; ceci encore est faiblement aux compétences pédagogiques après la formation et à leurs capacités à aider les étudiants et à utiliser proprement la technologie. En outre, les méthodes utilisées dans le cours de formation hybride telles : l'échange d'informations, les différentes méthodes de 
recherche et les activités des stagiaires sur Internet contribuent à accroître leurs capacités technologiques.

Pour déterminer l'effet des années d'expérience des enseignants sur les domaines d'études, nous avons effectué le test ANOVA afin de comparer les moyennes pour les années d'expérience des enseignants.

\begin{tabular}{|c|c|c|c|c|c|c|}
\hline & Experience & $\mathbf{N}$ & Mean & Std. Deviation & Sig. & \\
\hline \multirow{4}{*}{$\begin{array}{l}\text { Niveau de } \\
\text { maîtrise }\end{array}$} & Moins que 10 ans & 34 & 21.5 & 9.0 & \multirow{4}{*}{0.102} & \multirow{4}{*}{$\begin{array}{c}\text { Pas } \\
\text { Significatif }\end{array}$} \\
\hline & Entre 10 et 20 ans & 23 & 20.3 & 8.9 & & \\
\hline & Plus que 20 ans & 12 & 15.3 & 5.9 & & \\
\hline & Total & 69 & 20.0 & 8.7 & & \\
\hline \multirow{4}{*}{ Activités } & Moins que 10 ans & 34 & 23.9 & 8.9 & \multirow{4}{*}{0.026} & \multirow{4}{*}{ Significatif } \\
\hline & Entre 10 et 20 ans & 23 & 20.0 & 10.3 & & \\
\hline & Plus que 20 ans & 12 & 15.2 & 10.7 & & \\
\hline & Total & 69 & 21.1 & 10.1 & & \\
\hline \multirow{4}{*}{$\begin{array}{l}\text { Rechercher } \\
\text { de } \\
\text { l'information }\end{array}$} & Moins que 10 ans & 34 & 11.2 & 4.7 & \multirow{4}{*}{0.334} & \multirow{4}{*}{$\begin{array}{c}\text { Pas } \\
\text { Significatif }\end{array}$} \\
\hline & Entre 10 et 20 ans & 23 & 11.6 & 3.3 & & \\
\hline & Plus que 20 ans & 12 & 9.4 & 4.4 & & \\
\hline & Total & 69 & 11.0 & 4.3 & & \\
\hline \multirow{4}{*}{$\begin{array}{l}\text { Diffuser de } \\
\text { l'information }\end{array}$} & Moins que 10 ans & 34 & 15.0 & 6.0 & \multirow{4}{*}{0.033} & \multirow{4}{*}{ Significatif } \\
\hline & Entre 10 et 20 ans & 23 & 15.5 & 3.6 & & \\
\hline & Plus que 20 ans & 12 & 10.7 & 6.1 & & \\
\hline & Total & 69 & 14.4 & 5.5 & & \\
\hline \multirow{4}{*}{$\begin{array}{l}\text { Compétences } \\
\text { acquises }\end{array}$} & Moins que 10 ans & 34 & 24.8 & 7.3 & \multirow{4}{*}{0.488} & \multirow{4}{*}{$\begin{array}{c}\text { Pas } \\
\text { Significatif }\end{array}$} \\
\hline & Entre 10 et 20 ans & 23 & 25.4 & 3.9 & & \\
\hline & Plus que 20 ans & 12 & 22.6 & 8.8 & & \\
\hline & Total & 69 & 24.6 & 6.7 & & \\
\hline \multirow{4}{*}{ Obstacles } & Moins que 10 ans & 34 & 8.3 & 4.4 & \multirow{4}{*}{0.657} & \multirow{4}{*}{$\begin{array}{c}\text { Pas } \\
\text { Significatif }\end{array}$} \\
\hline & Entre 10 et 20 ans & 23 & 7.5 & 3.2 & & \\
\hline & Plus que 20 ans & 12 & 7.3 & 4.8 & & \\
\hline & Total & 69 & 7.8 & 4.1 & & \\
\hline \multirow{4}{*}{ Capacité } & Moins que 10 ans & 34 & 20.0 & 5.8 & \multirow{4}{*}{0.045} & \multirow{4}{*}{ Significatif } \\
\hline & Entre 10 et 20 ans & 23 & 17.0 & 7.4 & & \\
\hline & Plus que 20 ans & 12 & 14.6 & 8.2 & & \\
\hline & Total & 69 & 18.0 & 7.0 & & \\
\hline
\end{tabular}

- Il existe des différences statistiquement significatives dans l'axe « Activités » en fonction des années d'expérience; en comparant les moyennes arithmétiques, on constate que la moyenne la plus grande était pour la tranche d'enseignants qui ont des années d'expérience de moins de 10 ans. En d'autres 
termes, ce groupe d'enseignants pratique plus d'activités technologiques que les autres.

- Il existe des différences statistiquement significatives dans l'axe «Diffuser l'information » (dans les travaux, cours et projets...) selon les années d'expérience; en comparant les moyennes arithmétiques, nous constatons que le plus grand taux était pour les enseignants ayant moins de 20 ans d'expérience (Moins que 10 ans et Entre 10 et 20 ans). En d'autres termes, ces deux groupes d'enseignants ont la capacité d'employer et d'utiliser la technologie acquise pendant la formation universitaire plus que les enseignants expérimentés de plus de 20 ans.

- Il existe des différences statistiquement significatives dans l'axe « Capacité » en fonction des années d'expérience ; en comparant les moyennes arithmétiques, nous constatons que la moyenne la plus grande était pour la tranche d'enseignants qui ont des années d'expérience moins de 10 ans. En d'autres termes, les enseignants de cette classe sont plus capables d'aider et d'encourager les étudiants et de résoudre les problèmes techniques que les autres.

\section{Conclusion}

\subsection{Utilisations de la technologie}

L'utilisation de la technologie dans les salles de classe est à double tranchant : d'une part c'est une pratique qui attire les enseignants et d'autre part cette pratique cause une frustration. Quant au niveau de maîtrise de l'utilisation des technologies et leur intégration en classe, ceci varie selon les caractéristiques personnelles de l'enseignant surtout si les enseignants sont expérimentés ou selon leur tranche d'âge. En outre, les méthodes utilisées dans le cours de formation hybride telles : l'échange d'informations, les différentes méthodes de recherche et les activités des stagiaires sur Internet contribuent à accroître leurs capacités technologiques.

Les enseignants qui ont moins que 10 années d'expérience pratiquent plus d'activités technologiques que les autres, se sentent plus capables d'appliquer les compétences acquises dans leurs classes, ainsi que d'aider et d'encourager les étudiants et de résoudre les problèmes techniques que les autres.

L'étude a montré que certains utilisent la technologie comme un outil et un catalyseur supplémentaire pour les aider à compléter leur mission, en fournissant un espace plus large pour développer les relations avec les étudiants et être proche des besoins individuels de chacun. D'autres ne sont pas attachés à jeter le «nouvel invité» dans le système éducatif traditionnel ; ce qui explique l'intérêt croissant pour l'apprentissage mixte en abordant le changement du rôle des apprenants dans le processus d'apprentissage. Cette transformation a lieu lorsque le statut des apprenants affiche un changement 
de récepteurs passifs de connaissances à des constructeurs de connaissances actives. L'étude a encore révélé que les approches d'intégration avant la formation sont fortement influencées par les produits Microsoft parallèlement aux efforts du gouvernement dans ce domaine.

Guangying (2014) interprète le degré croissant d'attention accordé à l'apprentissage mixte dans le domaine de l'éducation par la contribution de l'apprentissage mixte à l'apprentissage grâce à sa flexibilité, sa rapidité et son pouvoir accéléré. Outre les avantages apportés par l'apprentissage mixte, aborder la question de savoir ce que cela englobe pourrait faciliter la compréhension de l'apprentissage hybride.

Les participants ont noté que l'expérience d'apprentissage mixte (hybride) était complètement nouvelle pour eux. Les enseignants ont signalé cette approche suit l'enseignement contemporain ce qui provoque la motivation des apprenants et fait appel à différents styles d'apprentissage y compris l'apprentissage collaboratif, continu, et autonome.

La fusion des environnements « en ligne » et en « face- à - face » dans l'apprentissage mixte a offert un ensemble unique d'opportunités et de défis dans le processus de conception de cours. Bien que de nombreux facteurs interviennent dans la construction réussie du cours mixte, la définition de la philosophie d'enseignement et d'apprentissage au départ constitue une base solide pour le développement d'un cours de qualité. Les facteurs de réussite cruciaux lors de l'élaboration des cours hybride impliquent l'amalgame approprié des stratégies d'enseignement et d'apprentissage pour vous en tant que facilitateur de l'apprentissage, les apprenants et l'environnement d'apprentissage.

\section{Considérations logistiques}

La grande majorité des enseignants avaient accès à un ordinateur et à Internet à domicile et au travail; de même, plus que la moitié des enseignants utilisent le tableau blanc interactif, les tablettes et peu utilisent les caméras vidéo. La fréquence d'utilisation des TICE en classe était 2 fois par semaine en moyenne. Concernant l'alphabétisation numérique nécessaire pour établir l'apprentissage hybride, les enseignants gèrent bien le Word et PPt ainsi que la recherche sur Internet mais rares sont ceux qui utilisent les logiciels pour planifier des cours en ligne ou produire des documents numériques tels les vidéos. La technologie telle qu'elle est utilisée en classe est considérée être un outil d'enseignement mais pas un outil d'apprentissage ; en plus elle est moins utilisée comme moyen pour améliorer la communication.

Concernant la méthode d'instruction utilisée, essentiellement, la question à se poser est de savoir comment chaque objectif d'apprentissage spécifique peut être atteint, le plus efficacement possible. Ce que les enseignants ont apprécié dans le cours était une démonstration du concept en 
classe avant de le poster en ligne ; l'interaction doit être abordée dans la session de cours en « face-à-face » et poursuivie en ligne dans le chat forum.

\subsection{Perceptions vis-à-vis du cours de formation hybride Contenu}

Les enseignants signalaient l'importance d'avoir une influence sur le contenu du cours et pouvoir améliorer les activités. En plus de la couverture en termes de profondeur et d'étendue, ce qui était important pour les enseignants était le séquençage et la transition entre les parties en ligne et en face-à-face du cours mixte. D'après les enseignants, le format hybride de l'apprentissage n'a pas rendu le cours très exigeant et était bien conforme à leur style d'apprentissage.

Définir une stratégie d'interaction et d'évaluation dans le cours consistait à déterminer les types d'activités, les devoirs, les exercices interactifs et l'évaluation. Les enseignants ont trouvé que c'est important de fournir des exemples et de donner assez de chances pour mettre en pratique les connaissances et les compétences acquises ce qui leur permet de s'autoévaluer. Par exemple, regarder un segment vidéo avant le cours pour initier une discussion en classe. Les discussions en ligne peuvent être utilisées pour poursuivre une conversation que les étudiants ont trouvée très intéressante lors d'une séance de cours.

\section{Communication}

La communication et la rétroaction sont essentielles à la création d'une expérience d'apprentissage mixte (hybride) de qualité et satisfaisante. Le forum, espace chat, a permis une communication entre étudiants (enseignants) et professeur, une collaboration et une interaction entre les étudiants (enseignants), et la communication des attentes et des protocoles dès le début du cours. Les enseignants ont signalé l'importance du soutien continu fourni durant la communication.

\section{TPACK}

L'enseignement des compétences en TICE ne sert pas bien les enseignants en formation, car ils apprennent à utiliser les outils liés aux TIC sans pouvoir les utiliser efficacement pour promouvoir l'apprentissage des élèves. Pour devenir un enseignant intégrant les TIC, il faut aller au-delà des compétences en TIC et comprendre les relations complexes qui existent entre pédagogie, contenu et TIC. Par conséquent, un programme de formation des enseignants devrait fournir aux étudiants les connaissances, les compétences et l'expérience nécessaires pour intégrer efficacement les TIC dans leur pratique future, en tenant compte des interactions entre la pédagogie, le contenu et les TIC. 
Cette étude a confirmé les conclusions des études précédentes que l'environnement d'apprentissage hybride a un effet positif sur le développement de TPACK des enseignants. Nous avons constaté que les étudiants ont l'impression que la formation hybride a conduit au développement de tous les domaines liés à TPACK.

\section{Quoi éviter}

Il y a cinq erreurs courantes commises par les enseignants lors de l'utilisation de la technologie:

Utiliser la technologie comme une alternative à une autre activité sans aucune amélioration dans la façon dont l'activité est réalisée, comme permettre aux étudiants de soumettre des recherches et des rapports imprimés via des ordinateurs non manuscrits.

Utiliser la technologie comme un ajout qui permet aux élèves d'utiliser des machines, Internet, des programmes électroniques ou des applications comme récompenses pour le divertissement ou pour remplir le temps libre de ceux qui terminent leur travail avant la fin de la leçon.

L'éducation technologique tel qu'elle n'est pas traitée comme un moyen d'enseignement et d'apprentissage mais comme une matière séparée.

La présence formelle de la technologie de l'information étant donné que la présence de la technologie en classe ne garantit pas sa bonne utilisation en tant que méthode ou stratégie pour faciliter le processus d'apprentissage.

Utiliser la technologie pour redynamiser les élèves et les encourager à apprendre. L'hypothèse que l'utilisation de la technologie peut être une solution au problème du manque de motivation pour les étudiants à apprendre est erronée. La motivation n'est pas synonyme d'apprentissage en l'absence d'objectifs pédagogiques clairs et forts qui établissent l'utilisation de la technologie.

\section{References:}

1. Alebaikan, R., \& Troudi, S. (2010). Blended learning in Saudi universities: challenges and perspectives. ALT-J Research in Learning Technology, 18 (1), 49-59. Retrieved online from: http://dx.doi.org/10.1080/09687761003657614

2. Bang, E., \& Luft, J. (2013). Secondary science teachers' use of technology in the classroom during their first 5 years. Journal of Digital Learning in Teacher Education, 29, 118- 126.

3. Barnum, C. \& Paarmann, W. (2002). Bringing induction to the teacher. A Blended learning model. T.H.E. Journal, (30), 2, 56-64.

4. Chai, C.S., Koh, J. H.L., \& Tsai, C.C. (2013). A Review of Technological Pedagogical Content Knowledge. Educational Technology \& Society, 16 (2), 31-51. 
5. Chien, Y.T., Chang, C.Y., Yeh, T.K., \& Chang, K.E. (2012). Engaging pre-service science teachers to act as active designers of technology integration: A MAGDAIRE framework. Teaching \& Teacher Education, 28, 578-588.

6. Davies, R. S., Dean, D. L., \& Ball, N. (2013). Flipping the classroom and instructional technology integration in a college-level information systems spreadsheet course. Educational Technology Research and Development, 61(4), 563-580. doi: 10.1007/s11423-013-9305-6.

7. Diab, H. (2014). Documentary of events during Minister Hassan Diab's term at the Ministry of Education \& Higher Education. Beirut: Typopress.

8. Dziuban, C. D., Hartman, J.L. \& Moskal, P.D. (2010). Blended Learning, ECAR Research Bulletin, 7, Retrieved online, from http://www.educause.edu/ir/library/pdf/ERB0407.pdf.

9. Gao, J. P., Wong, A. F., Choy, D., \& Wu, J. (2011). Beginning teachers' understanding performances of technology integration. Asia Pacific Journal of Education, 31, 211-223.

10. Garrison, D.R., \& Kanuka, H. (2004). Blended learning: Uncovering its transformative potential in higher education. The Internet and Higher Education, 7 (2), 95-105.

11. Gronseth, S., Brush, T., Ottenbreit-Leftwich, A., Strycker, J., Abaci, S., Easterling, W., Roman, T., Shin, S., \& van Leusen, P. (2010). Equipping the next generation of teachers: Technology preparation and practice. Journal of Digital Learning in Teacher Education, 27(1), 3036.

12. Finger, G., Albion, P., Jamieson-Proctor, R., Cavanagh, R., Grimbeek, P., Lloyd, M., Fitzgerald, R., Bond, T, \& Romeo, G. (2013). Teaching Teachers for the Future (TTF) Project TPACK Survey: Summary of the Key Findings. Australian Educational Computing, Special Edition: Teaching Teachers for the Future Project. 27(3), 13-25.

13. Magner, T., Soulé, H., \& Wesolowski, K. (2011). P21 Common Core toolkit: A guide to aligning the Common Core State Standards with the Framework for 21st Century Skills. Washington, DC: Partnership for 21st Century Skills. Retrieved from www.p21.org/storage/documents/P21CommonCoreToolkit.pdf.

14. Ministry of Education and Higher Education (MEHE). (2012). Teaching and learning in the digital age: Lebanon's National Educational Technology Strategic Plan. The Ministry of Education and Higher Education Strategic Planning Development Team, Beirut, Lebanon. Retrieved December 10, 2017, from http://www.mehe.gov.lb/Uploads/file/TLSP.pdf 
15. Mishra, P., \& Koehler, M. J. (2006). Technological Pedagogical Content Knowledge: A new framework for teacher knowledge. Teachers College Record, 108 (6), 1017-1054.

16. Pamuk, S. (2011). Understanding preservice teachers' technology use through TPACK framework. Journal of Computer Assisted Learning. Advance online publication. doi: 10.1111/j.1365-2729.2011.00447.

17. Sweeney, T., \& Drummond, A. (2013). How Prepared are our Pre-service Teachers to Integrate Technology? A Pilot Study. Australian Educational Computing, Special Edition: Teaching Teachers for the Future Project, 27(3), 117-123.

18. Tondeur, J., Van Braak, J., Sang, G., Voogt, J., Fisser, P. \& OttenbreitLeftwich, A. (2012). Preparing Pre-Service Teachers to Integrate Technology in Education: A Synthesis of Qualitative Evidence. Computers \& Education, 59(1), 134-144. Retrieved June 24, 2018 from https://www.learntechlib.org/p/67082/.

19. Vaughan, N. (2007). Perspectives on Blended Learning in Higher Education: International Journal on E-Learning. 6 (1), 81-94. Chesapeake, VA: AACE.

20. Wetzel, K., Buss, R. Foulger, T.S. \& Lindsey, L.A. (2014). Infusing Educational Technology in Teaching Methods Courses: Successes and Dilemmas. Journal of Digital Learning in Teacher Education, 30(3), 89-103, DOI: 10.1080/21532974.2014.891877

21. Williams, C. (2002). Learning on-line: A review of recent literature in a rapidly expanding field. Journal of Further and Higher Education, 26(3), 263-272. 